\title{
ARTICLE OPEN \\ Manifold learning of four-dimensional scanning transmission electron microscopy
}

\author{
Xin $\mathrm{Li}^{1,2}$, Ondrej E. Dyck ${ }^{1,2}$, Mark P. Oxley ${ }^{1,2}$, Andrew R. Lupini ${ }^{1,2}$, Leland Mclnnes $\mathbb{D}^{3}$, John Healy ${ }^{3}$, Stephen Jesse ${ }^{1,2}$ and \\ Sergei V. Kalinin ${ }^{1,2}$
}

Four-dimensional scanning transmission electron microscopy (4D-STEM) of local atomic diffraction patterns is emerging as a powerful technique for probing intricate details of atomic structure and atomic electric fields. However, efficient processing and interpretation of large volumes of data remain challenging, especially for two-dimensional or light materials because the diffraction signal recorded on the pixelated arrays is weak. Here we employ data-driven manifold leaning approaches for straightforward visualization and exploration analysis of 4D-STEM datasets, distilling real-space neighboring effects on atomically resolved deflection patterns from single-layer graphene, with single dopant atoms, as recorded on a pixelated detector. These extracted patterns relate to both individual atom sites and sublattice structures, effectively discriminating single dopant anomalies via multimode views. We believe manifold learning analysis will accelerate physics discoveries coupled between data-rich imaging mechanisms and materials such as ferroelectric, topological spin, and van der Waals heterostructures.

npj Computational Materials (2019)5:5; https://doi.org/10.1038/s41524-018-0139-y

\section{INTRODUCTION}

Over the past decade, Scanning Transmission Electron Microscopy (STEM) has seen a growing trend towards full capture of the information stream including amongst many others, threedimensional (3D) electron tomography, ${ }^{1}$ four-dimensional (4D) ptychography, ${ }^{2} 4 \mathrm{D}$ phase plate STEM $^{3}$, and five-dimensional (5D) in-situ beam electron diffraction. ${ }^{4}$ Efficient phase contrast imaging in STEM using a pixelated detector provides comprehensive information at each scanning location via the Ronchigram images 5 (In the rest of paper, we use the notation of Ronchigram to indicate the zero order disk from a beam diffraction pattern recorded on a pixelated detector). A large body of theoretical and experimental work has been conducted, revealing that $4 D$ Ronchigram datasets enable, for example, super resolution ${ }^{6,7}$ and three-dimensional imaging. ${ }^{8-10}$ Aside from efficient phase imaging, Pennycook et al..$^{5}$ concluded that Ronchigram images should have greater sensitivity to electromagnetic fields than differential phase contrast imaging (DPC) which is currently considered as the state-of-the-art to directly visualize local electromagnetic fields. ${ }^{11-18}$ With recent progress in highsensitive area detectors, ${ }^{19}$ DPC has revealed atomic electric fields in crystal by both segmented detectors ${ }^{20,21}$ and pixelated detectors with a simplified quantum theoretical interpretation. ${ }^{22}$

Despite the above progress, a vast fraction of the information contained in the Ronchigram is currently left unutilized, due to the lack of understanding of how physical parameters such as instrumentation settings and material structures affect the deflection patterns (inhomogeneous electron intensity distribution recorded on the pixelated detector), necessitating a datadriven analytical framework without excessive prior domain knowledge. Here we investigate the deflection patterns and the associated real-space distributions over atomic structures via low- dimensional manifold learning of the Ronchgiram datasets. It is often the case that the underlying structure of the dataset as a whole can be described in terms of a much smaller number of latent features than the high dimensional set of measurements. The low-dimensional physical parameter space is translated onto a high dimensional response space by means of the imaging mechanisms. Aside from possible discontinuities due to material structure transitions and non-linear imaging transfer functions, points in close proximity in the physical parameter space will generally be in close proximity in the response space, forming a complex non-linear manifold. The non-linearity often renders linear dimension reduction methods ${ }^{23}$ of limited usefulness. Manifold learning is a set of well-established non-linear techniques in the machine learning community for finding such latent structure in high dimensional data.

Denote the collected Ronchigram datasets over a scanned area as $\mathbf{X}=\left\{\mathbf{X}_{1}, \mathbf{X}_{2}, \ldots . ., \mathbf{X}_{\mathbf{n}}\right\} \subseteq R^{p}$, where $n$ is the total number of scanned pixel locations and $p$ is the image size of an individual Ronchigram. We use the recently developed uniform manifold approximation and projection (UMAP ${ }^{24}$ ) method to represent the high-dimensional Ronchigram datasets via fuzzy topological sets with regard to the Riemannian metric. For straightforward visualization and exploration analysis, here UMAP optimizes the low-dimensional manifold representation $\mathbf{Y}=\left\{\mathbf{Y}_{\mathbf{1}}, \mathbf{Y}_{\mathbf{2}}, \ldots . .\right.$. , $\left.\mathbf{Y}_{\mathbf{n}}\right\} \subseteq R^{d}, d=2,3$ of the original Ronchigram datasets $\mathbf{X}$, by minimizing the cross entropy of the two fuzzy set representations for $\mathbf{X}$ and $\mathbf{Y}$. Efficient implementation of UMAP follows the work of LargeVis, ${ }^{25}$ by firstly constructing approximate nearest neighbor graph via random projection tree ${ }^{26}$ and neighbor exploring, ${ }^{27}$ then solving the low-dimensional manifold embedding via probabilistic edge sampling and negative sampling. ${ }^{28}$ Further analysis of the intrinsic structures within the manifold can be

\footnotetext{
${ }^{1}$ Center for Nanophase Materials Sciences, Oak Ridge National Laboratory, Oak Ridge, TN 37831, USA; ${ }^{2}$ Institute for Functional Imaging of Materials, Oak Ridge National Laboratory, Oak Ridge, TN 37831, USA and ${ }^{3}$ Tutte Institute for Mathematics and Computing, Ottawa, Canada

Correspondence: Sergei V. Kalinin (sergei2@ornl.gov)
}

Received: 20 July 2018 Accepted: 6 December 2018

Published online: 07 January 2019 


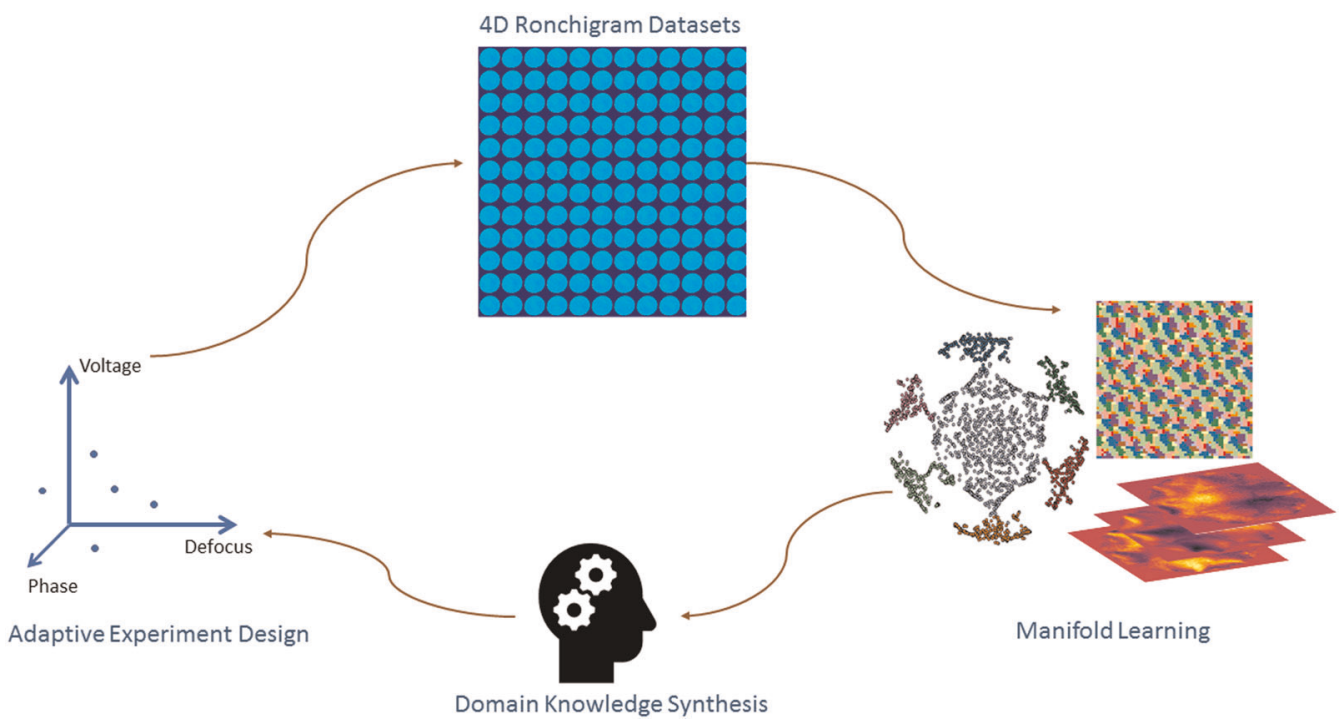

Fig. 1 Schematic of manifold learning of Ronchigram datasets. The low-dimensional physical parameter space (such as defocus, accelerating voltage, and material structure phases) is translated onto a high dimensional Ronchigram response space by the imaging mechanisms of microscope. High-dimensional and large-scale Ronchigram datasets are projected into a low-dimensional manifold space for efficiently revealing and hierarchically representing rich features. With extracted patterns from manifold learning, deeper study can be conducted via adaptive experiment design

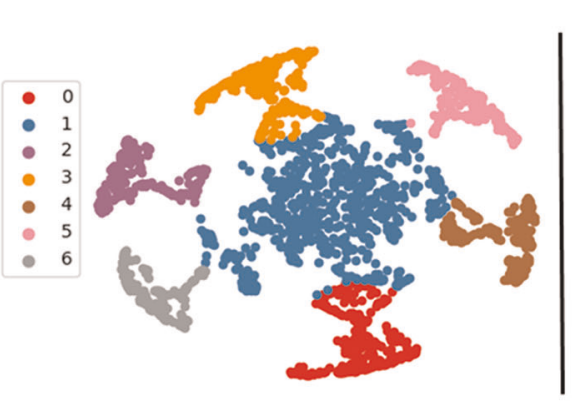

b

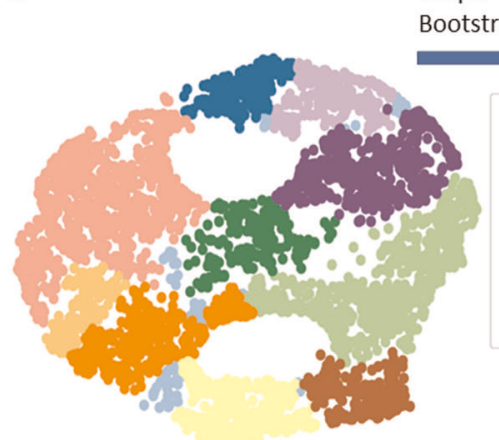

C

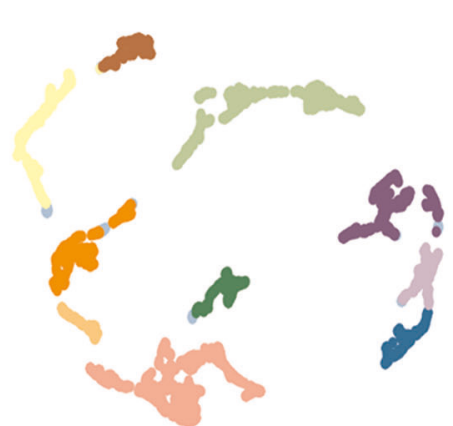

Fig. 2 Manifold bootstrapping and clustering. a Spectral clustering results on UMAP manifold derived from the synthetic dataset. b UMAP manifold and c Bootstrapped UMAP manifold derived from the experimental dataset. UMAP and bootstrapped UMAP manifolds are colored by the same set of clustering labels where HDBSCAN clustering was performed on the bootstrapped manifold. Bootstrapped manifold in $\mathbf{c}$ is derived from the reconstructed graph based on the $\mathbf{b}$ UMAP manifold

performed via the machine learning technique known as clustering. Trying to further separate manifold clusters and present them in a clearer way, Li et al. ${ }^{29}$ empirically proposed Graph-Bootstrapping that iteratively reconstructs the nearest neighbor graph based on previous manifold positions and then recalculates manifold coordinates based on the reconstructed graph.

Remarkably, although the proposed manifold learning framework is purely data-driven without any prior bias regarding the material structure and instrumental modality, it reveals the realspace neighboring effects on deflection patterns in the Ronchigram sampled over single-layer graphene with single dopant atoms. These deflection patterns relate to both individual atomic site and the sublattice structure and can be used to effectively discriminate dopant anomalies via multiple-mode views. For the experimental datasets of size $(64 \times 64,180 \times 180)$, the computation took $<10 \mathrm{~min}$ on a single workstation (Intel Xenon E5-1650V3, 32GB DDR3 RAM) to get the findings analyzed in this paper.

Figure 1 illustrates the flowchart of manifold-learning accelerated discovery of physics coupled between imaging mechanisms and material systems. High-dimensional Ronchigram datasets are projected into a low-dimensional manifold space for efficiently revealing and hierarchically representing rich features. With extracted patterns from manifold learning, deeper study can be conducted via adaptive experiment design. In addition, we believe this type of analysis could also stimulate the development of advanced machine learning algorithms. The driving goal of unsupervised learning is to discover unknown but interesting patterns within data. Due to the inherent absence of labels within the field of unsupervised machine learning, the ultimate validation of our work is the discovery of interesting, useful and externally validated results. External validation, such as on data with physical meaning, is an example of how we hope to develop mathematically sound and broadly applicable techniques, while simultaneously confirming the physical interpretations.

\section{RESULTS}

In this paper we focus on two specific example datasets, one synthetic dataset where the ground-truth input (the sample atomic positions and scattering factors) are known exactly, and an experimental dataset with several unknown parameters. The 
a

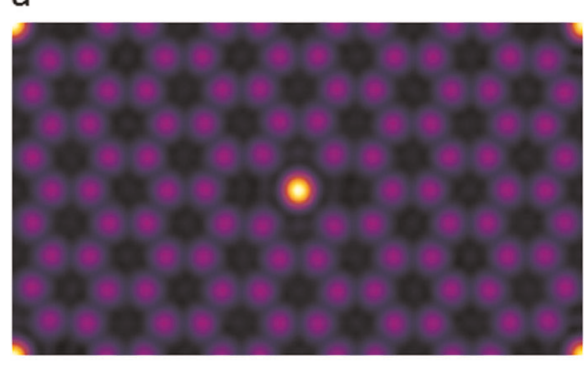

b

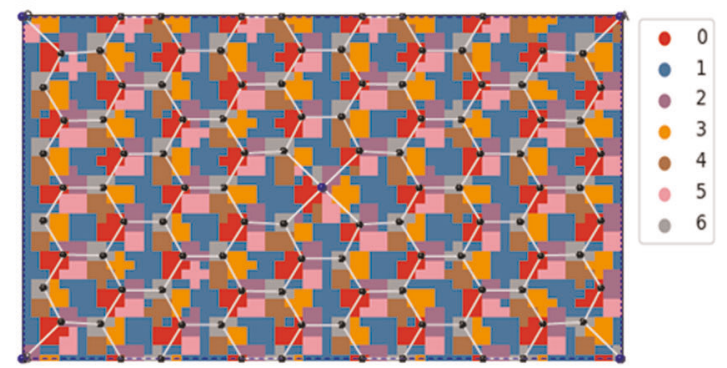

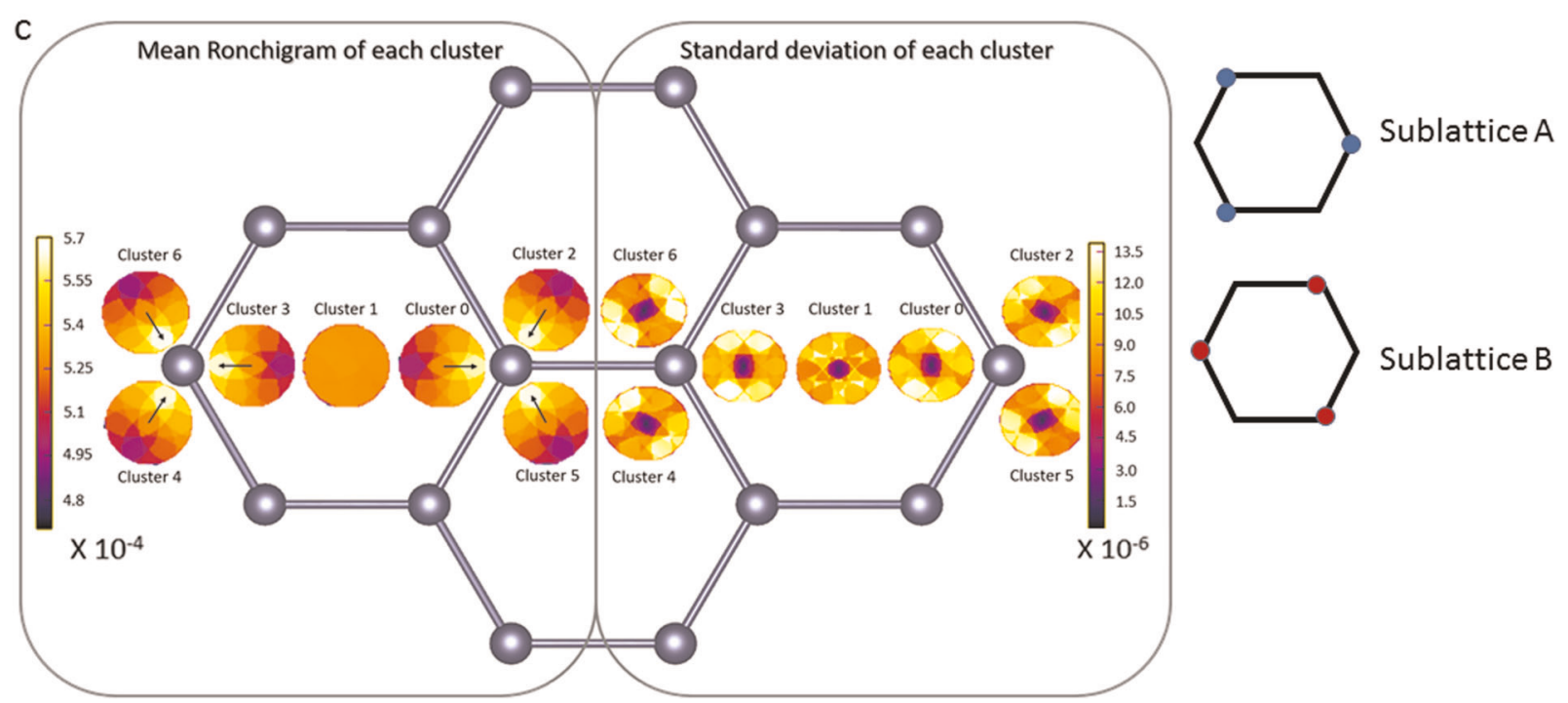

Fig. 3 Synthetic data analysis. a HAADF image of the synthetic dataset. b Ground-truth atom positions overlaid on real-space distributions of cluster labels in Fig. 2a. Black atoms are carbon, and blue ones are the Si dopants. c The mean and standard deviation (Std) of Ronchigrams for each cluster and positions to the atom sites. Arrows indicate the simulated beam deflection observed near an atom. The Ronchigram intensity moves toward the atom

simulated data is a $(37,64,120,140)$ synthetic dataset over $37 \times 64$ probe positions with the individual Ronchigram of size of $120 \times$ 140 pixels. The experiment consists of $64 \times 64$ probe positions with $180 \times 180$ Ronchigram pixels giving a $(64,64,180,180)$ experimental dataset.

\section{Manifold clustering}

In this work, we only consider the Euclidean distance as the distance metric. UMAP requires the tuning parameters of local neighborhood size and the effective minimum distance between embedded points, which we set to be 50 and 0 respectively for all manifold learning cases including the Graph-Bootstrapping procedure. We leave all the other tuning parameters of UMAP as default.

For the clean synthetic dataset, UMAP projects the graphene Ronchigram dataset into the shape of a hexagon in Fig. 2a. With a clear manifold layout, based on visual inspection on manifold shape, we can simply perform spectral clustering ${ }^{30}$ with 7 clusters. Supplementary Figure 1 contains manifold layouts from 25 reruns of UMAP on the synthetic dataset, where we overlay the same set of cluster labels in Fig. 2a.

Figure $2 \mathrm{~b}$ displays the UMAP manifold derived from the experimental dataset. We more often could not get a good guess on the number of clusters based on manifold shape due to noise and distortions associated in the experimental data (imagine Fig. 2b without color labels). To further extract the cluster structure, we calculate bootstrapped UMAP manifold by first reconstructing the graph from UMAP manifold (Fig. 2b) then recalculating the manifold embedding from the reconstructed graph as shown in Fig. 2c. Since the number of clusters is related to the structure-pattern relationships that is an unknown parameter we would like to estimate, clustering on manifold should be generally based on local structure such as nearest neighbor and density. Here we utilize the hierarchical density estimate methods (HDBSCAN ${ }^{31,32}$ ) to perform clustering on the bootstrapped UMAP manifold. Mathematically, HDBSCAN relies on the mutual reachability distance: $D_{\text {mreach, }}(a, b)=\max \left\{\operatorname{core}_{i}(a)\right.$, core $\left._{i}(b), \mathrm{d}(a, b)\right\}$, where $\mathrm{d}(a, b)$ is the original metric distance between points $a$ and $b, \operatorname{core}_{i}(x)$ is the core distance of a point $x$ to cover its ith nearest neighbor.

The primary tuning parameter of HDBSCAN is the minimum cluster size $k$. We follow a similar procedure in ref. ${ }^{29}$ to choose $k$. We first consider all integer $k$ values in the range $[20,149]$. In supplementary Figure 2, we then plot the trend of total number of estimated clusters against every $k$ value and fit this trend by the exponential decay. We choose the $k$ in the tail region where the total number of clusters tends to be stable. Specifically, we set $k=$ 86 in this case. We leave all the other tuning parameters of HDBSCAN as default.

HDBSCAN clustering is performed on bootstrapped UMAP manifold. Figure $2 \mathrm{~b}, \mathrm{c}$ show the clustering patterns over UMAP and bootstrapped UMAP manifolds with the same set of HDBSCAN cluster labels. We note that points with label of " -1 " are the "outliers" identified by HDBSCAN that do not belong to any cluster. Supplementary Figure 3 contains manifold layouts from 25 reruns of UMAP on the experimental dataset, where we overlay the same set of cluster labels in Fig. 2b, c. 


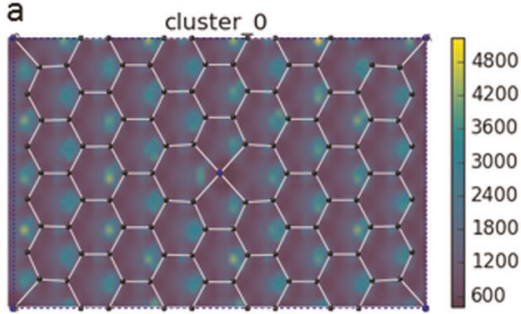

d

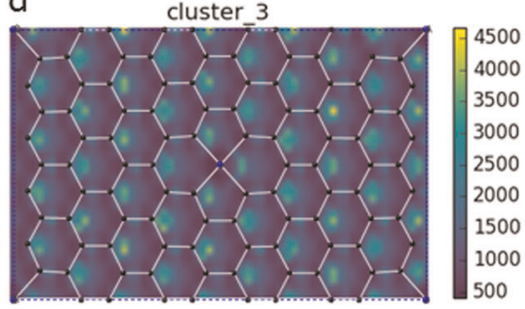

b

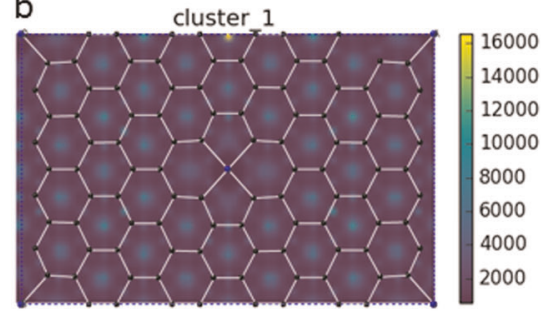

e

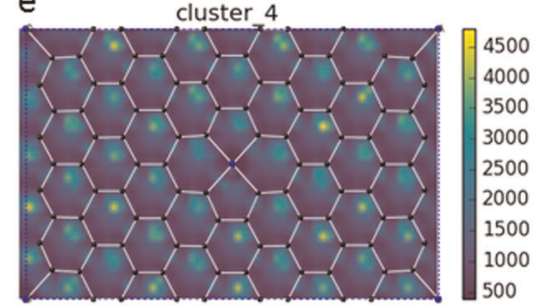

C
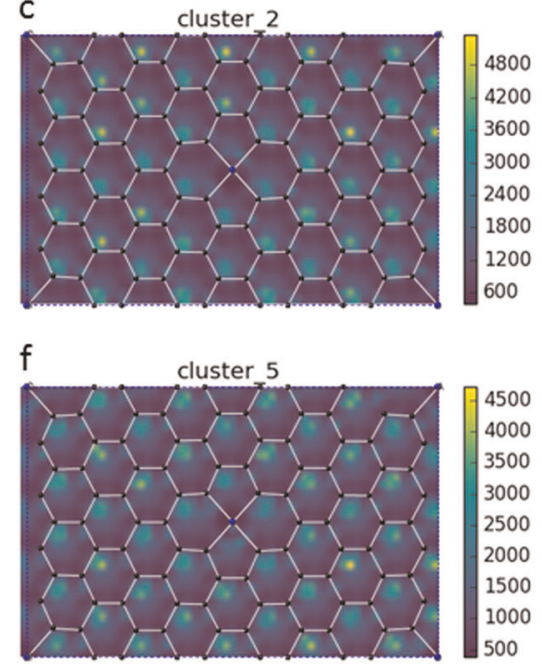

g

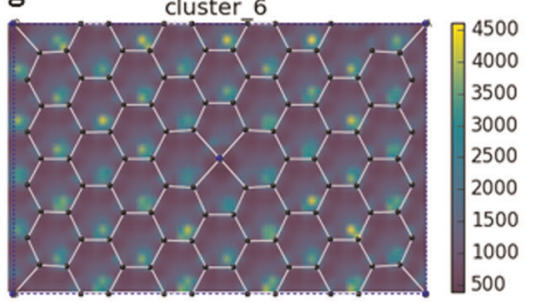

Fig. 4 Similarity loadings of clusters from the synthetic dataset. $\mathbf{a}, \mathbf{c}$, f Similarity loadings of clusters $0,2,5$ over sublattice A. b Similarity loadings of cluster 1 located in the space between atoms. d, e, $\mathbf{g}$ Similarity loadings of clusters 3,4,6 over sublattice B

a

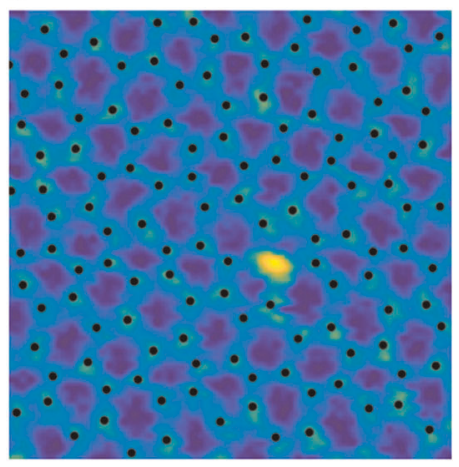

b

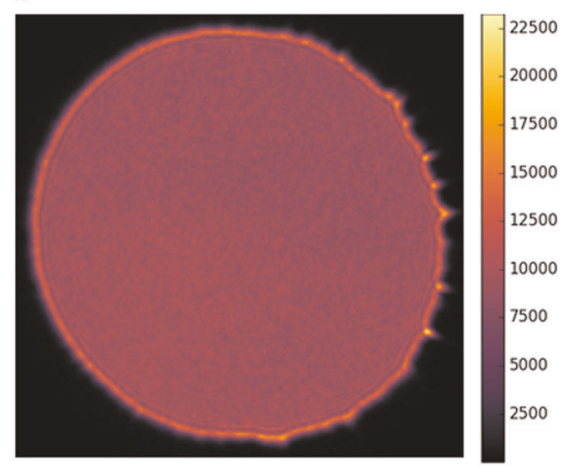

C

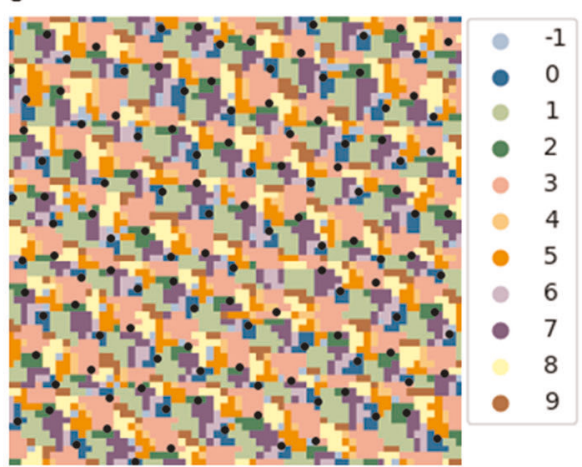

Fig. 5 Experimental data analysis. a The HAADF image overlaid with atom positions. b An as-acquired experimental Ronchigram image, corresponding to the top-left pixel position in a. c Real-space distributions of cluster labels in Fig. 2c

Synthetic dataset analysis

Figure $3 a$ is the high-angle annular dark filed (HAADF) image of the synthetic dataset. Figure $3 \mathrm{~b}$ displays the known, ground-truth atom positions overlaid on the spatial mapping of cluster labels derived from the manifold space in Fig. 2a. There are 5 Si dopants with one 4-fold coordinated dopant located in the middle and the others located at the four corners. Most obviously, we can see clusters $0,2,5$ and clusters 3,4,6 consist of two groups that are located around atom sites on the two sublattices of graphene respectively, while cluster 1 is mostly located in the space between atoms. Figure $3 c$ displays the mean and standard deviation (Std) of Ronchigrams for each cluster with positions to the atom sites. We note that standard deviations $\left(10^{-6}-10^{-5}\right)$ are one magnitude smaller than mean values $\left(10^{-4}\right)$, showing the clustering accuracy. Arrows in Fig. $3 \mathrm{c}$ are used to indicate that the electron beam is deflected towards the direction of atom nuclei which is consistent with previous work on unveiling atomic electric fields with DPC. ${ }^{21,22}$ From Fig. 3b, c, we further note that Ronchigrams with opposite deflection patterns are located at opposite sides of the two mirrored atom sites over the two sublattices, instead of the two sides of the same atom, revealing the real-space neighboring effects on deflection patterns. It is also worthwhile to point out the irregular real-space position arrangements of cluster labels located at Si dopants.

To check the above finding quantitatively, one can calculate the similarity loadings by calculating pairwise distances between the mean Ronchigram of the cluster and every individual Ronchigram. Figure 4 shows the similarity loadings (inverse of pairwise Euclidean distances) of clusters. For every cluster, the distribution of bright blobs in the similarity loading is consistent with cluster label positions. Comparing similarity loadings of clusters with 


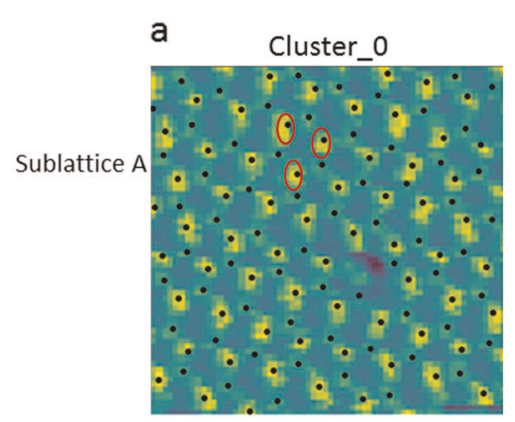

b

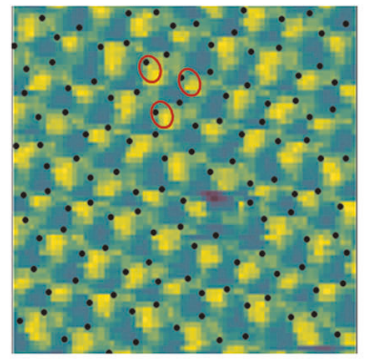

C
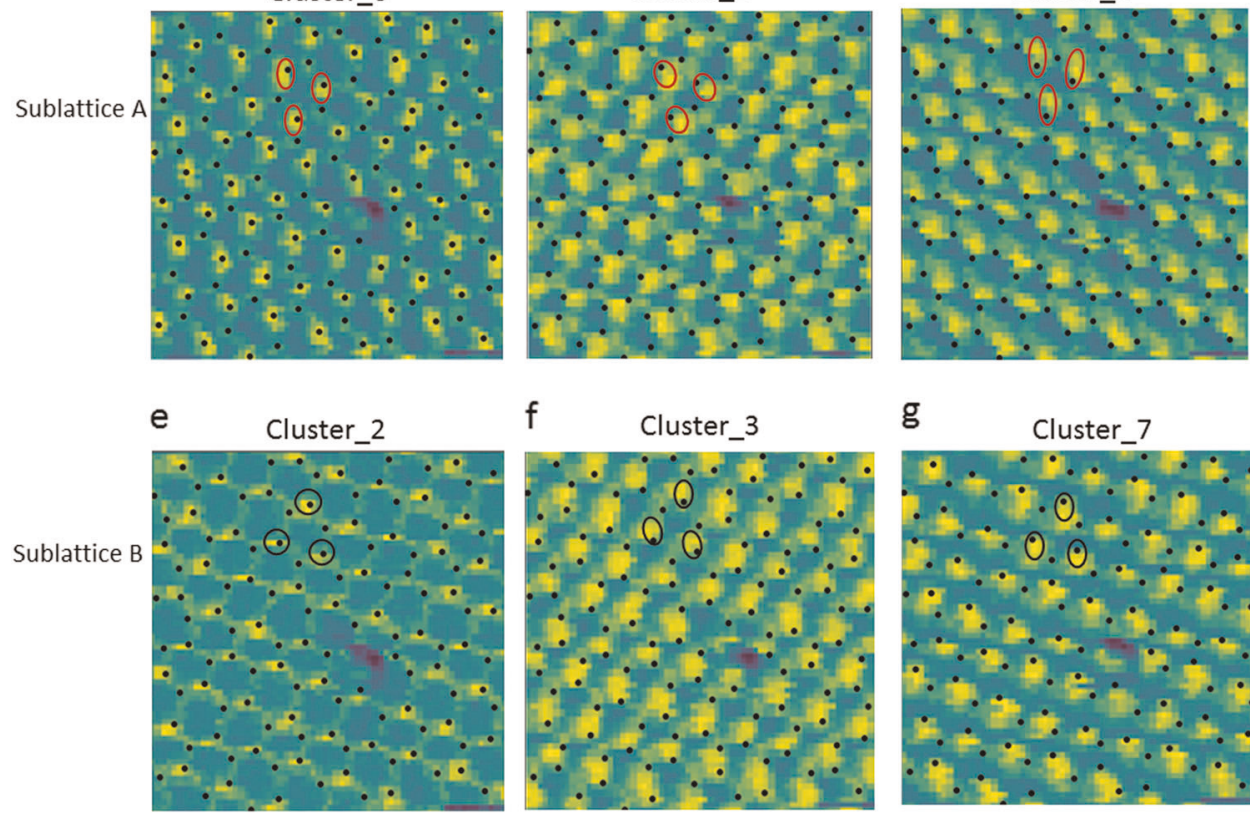

f Cluster_3

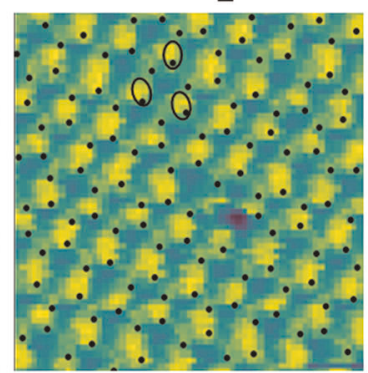

g

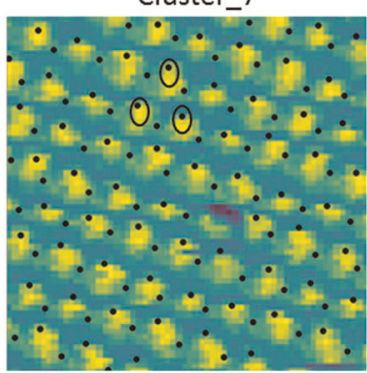

d

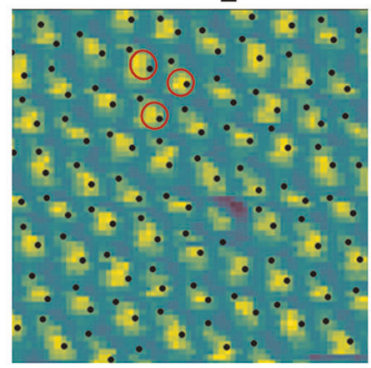

h

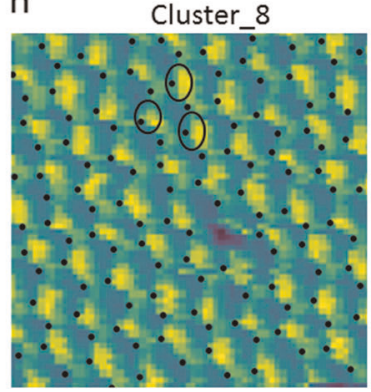

Fig. 6 Similarity loadings of clusters from the experimental dataset. a-d Similarity loadings of clusters over sublattice a. e-h Similarity loadings of clusters over sublattice $\mathbf{b}$. Here we overlay the circle markers to illustrate the relative positions between atom sites and bright blobs
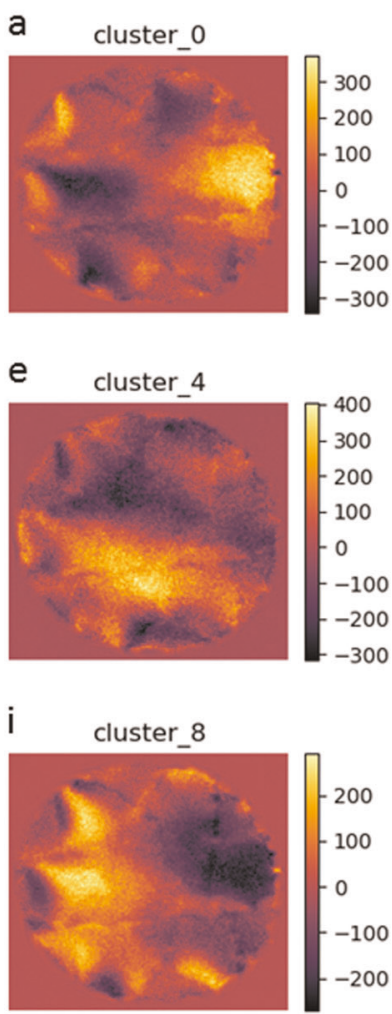
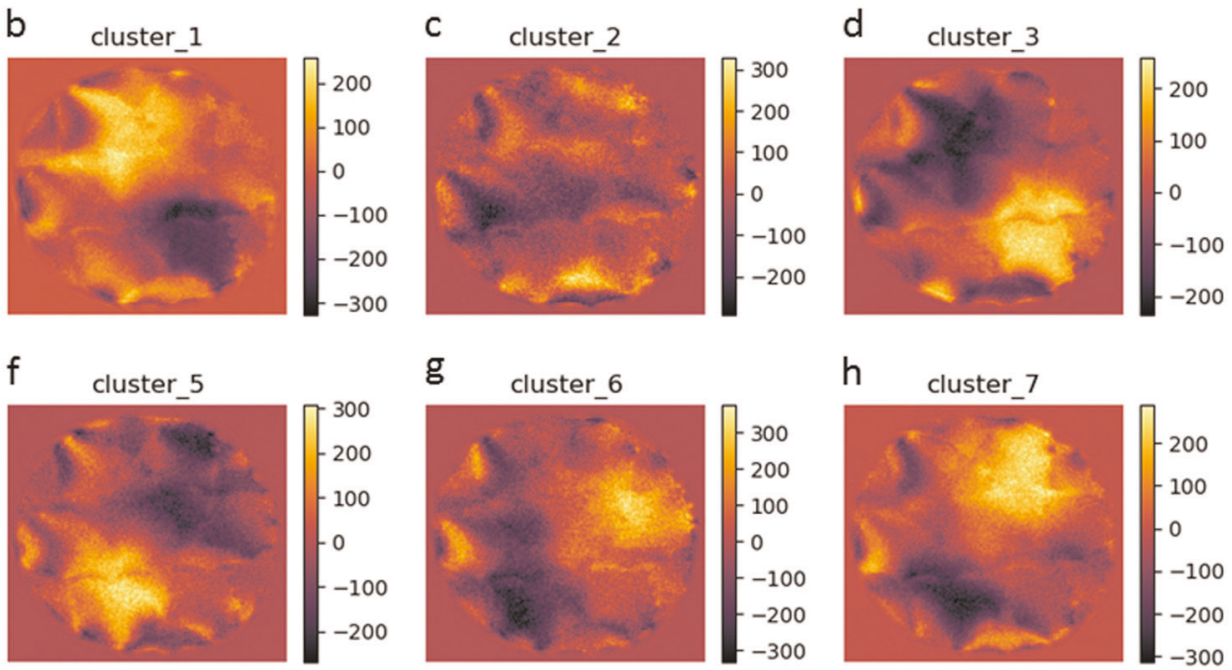

$\mathrm{h}$

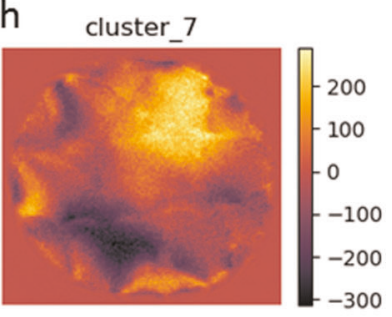

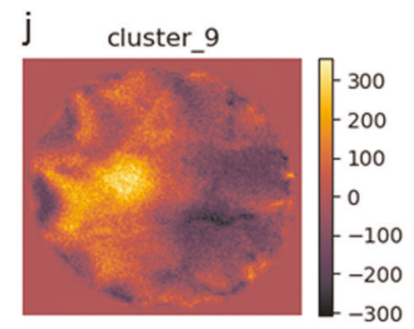

Fig. 7 Deflection patterns in cluster Ronchigrams from the experimental dataset. a-j Deflection patterns in mean Ronchigrams for clusters $0-9$. Here we subtracted the mean of all Ronchigrams from mean Ronchigram of each cluster

opposite deflection patterns in Fig. 4 (clusters 0 and 3, clusters 2 and 4 , clusters 5 and 6), we see none of those bright blobs are located at opposite sides of the same atom site. Instead, they are located at the opposite sides of the two mirrored atom sites over sublattices. Effectively, the method decomposes deflection patterns into 2 classes that are spatially related to the sublattices, instead of being radially symmetric around each atom site. We conjecture that both individual atom site and its local neighbor atoms will affect the deflection pattern. Furthermore, we notice that all similarity loadings display a black patch at the 4-fold Si 
a

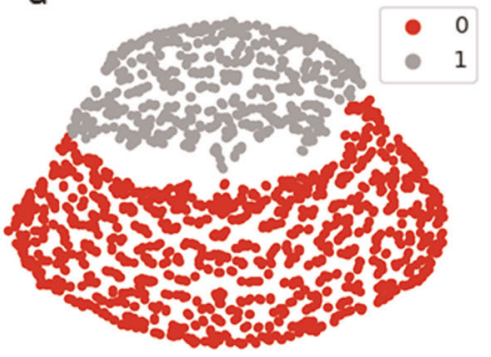

d

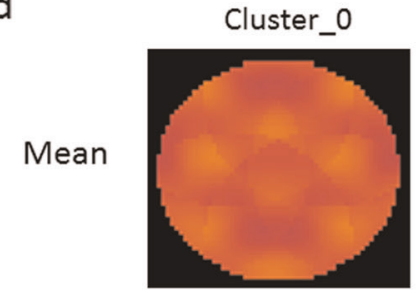

Cluster_0

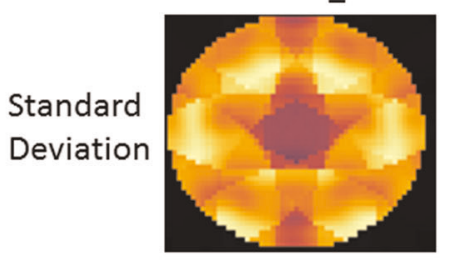

$b$
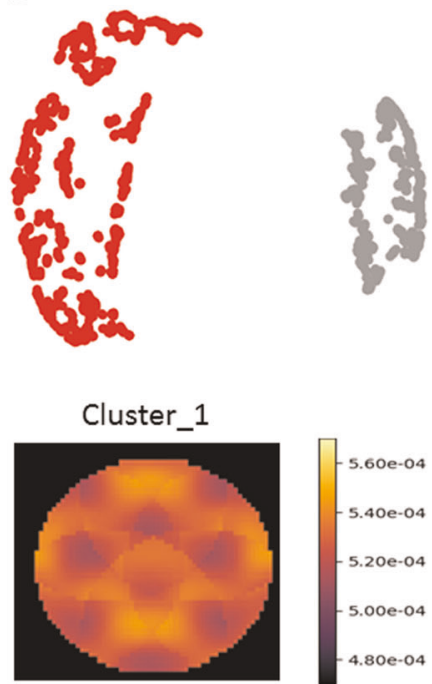

Cluster_1

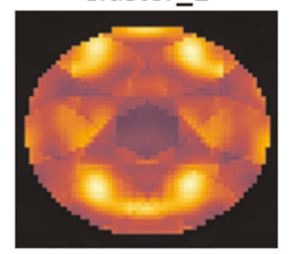

C

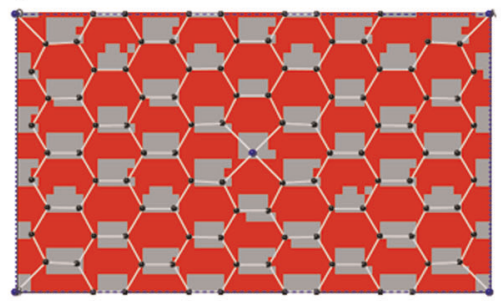

e

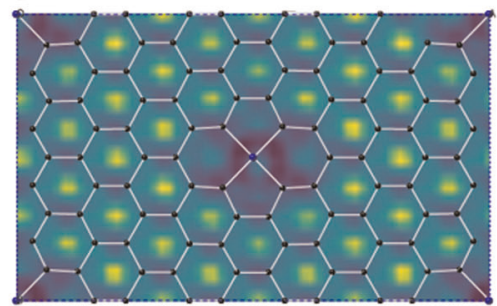

Cluster_0

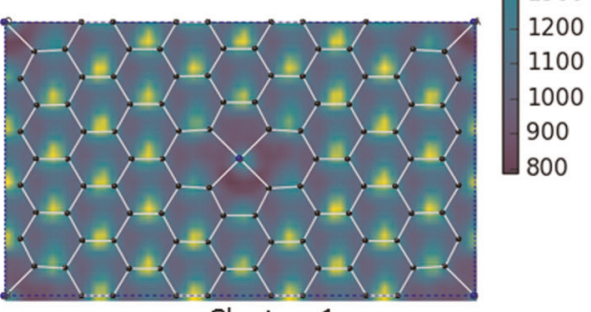

Cluster 1

Fig. 8 Synthetic data analysis with defocus setting. a UMAP and b bootstrapped UMAP manifolds. $\mathbf{c}$ Real-space distributions of cluster labels. d The mean and standard deviation (Std) of Ronchigram for each cluster. e Similarity loadings of the clusters

dopant position in the middle, indicating the deflection patterns around the Si dopant are significantly different from those around the carbon atom.

\section{Experimental dataset analysis}

Figure 5a shows the HAADF image of graphene with a single 4fold silicon dopant, overlaid with estimated atom positions and Fig. $5 \mathrm{~b}$ is an example of an as-acquired experimental Ronchigram where the deflection pattern is weak due to the monolayer structure of graphene. Nonetheless manifold learning could still distinguish intrinsic patterns in the real dataset without any signal pre-processing. Figure $5 \mathrm{c}$ displays the spatial distribution of cluster labels showing the sublattice structures.

Figure 6 shows the similarity loadings of the clusters. Based on the relative positions between atom sites and bright blobs as illustrated by the circle markers, we categorize clusters into sublattice A (clusters $0,1,5,6$ ) and sublattice B (clusters 2,3,7,8). Again, all the similarity loadings display a black patch at the position of the 4-fold Silicon dopant, implying differences between Ronchigrams sampled around the Si dopant and those around the carbon atom. Supplementary Figure 4 shows the similarity loadings of clusters (clusters 4,9) whose spatial locations are not indicative to either sublattice.

Finally, trying to see the relative patterns in experimental Ronchigrams, we subtracted the mean of all Ronchigrams from the mean Ronchigram of each cluster in Fig. 7. Supplementary 5 displays the original mean Ronchigrams of clusters with a manually tuned range of colorbar. By referring to the same cluster number, we can verify that deflection patterns in cluster Ronchigrams (Fig. 7, Supplementary Figure 5) are consistent with associated cluster label positions to the atom sites as indicated in
Figs. 5c and 6 and Supplementary Figure 4. Supplementary Figure 6 provides a compact illustration of deflection patterns in Ronchigrams and associated positions to the atom sites.

\section{DISCUSSION}

Over the past 10 years, several groups have made significant contributions towards visualization of electromagnetic fields via segmented and pixelated detectors. However, at the present time, a vast fraction of the information contained in the Ronchigram is still not fully exploited and there remains a lack in knowledge of how physical parameters affect the electron distribution on pixelated detectors. Here we utilize the data-driven manifold learning methodology, to spatially map the local inhomogeneities captured in the Ronchigram datasets and to do so without requiring a priori model or placing any constraints on the data. This machine learning method could provide a fast screening tool for domain experts to digest the Ronchigram patterns at large scale and build knowledge libraries of various effects made on Ronchigram. To illustrate the above point, Fig. 8 shows the Ronchigram patterns from the synthetic dataset with $-10 \mathrm{~nm}$ defocus. Otherwise, the defocused dataset was calculated as described in the methodology section. Under this defocus setting, the manifolds in Fig. 8a, b only consist of two groups. The spatial distributions of cluster labels are no longer indicative of the graphene sublattice structure. To study the patterns of Ronchigram, attention should be paid on design of experiment, with careful control and recording of the experimental conditions.

We have shown that manifold learning is a highly efficient method to process and visualize large scale 4D-STEM datasets of atomically resolved diffraction patterns. As we highlight in both the synthetic and experimental data analysis, even though 
diffraction pattern is very weak due to the monolayer structure of graphene, we can distill hidden information from Ronchigrams. Instead of the radial symmetry centered at each atom site, manifold learning analysis separates the Ronchigrams based on local symmetry dependent on the sublattices, indicating the realspace neighboring effects on deflection patterns. We propose that manifold learning and other related techniques will be extremely promising routes for analyzing the wealth of multidimensional data emerging from a new generation of electron microscopes, to denoise, interpolate, and explore the data that will be of interest to many fields such as ferroelectric, topological spin, and van der Waals heterostructures.

In particular, this approach may open a pathway for low dose imaging and effective algorithms for atomic manipulations $s^{33-37}$ that currently rely on sequential imaging and probe positioning. Methods based on the Ronchigram will have the potential for more efficient use of the signal for thin samples than the readily interpretable HAADF image,${ }^{5}$ effectively allowing us to use a single point signal as a proxy for the local structure and obviate the need for sub-scan or Fourier transform based feedback. ${ }^{38}$ Efficient manifold learning method might be adapted to provide a fast measure of whether a certain area matches a known cluster or represents an anomaly, with potential benefits for real-time feedback and control.

\section{METHODS}

Simulation of Ronchigrams was carried out using the quantum excitation of phonons option in the $\mu$ STEM package. ${ }^{39}$ Calculations were carried out on a $768 \times 768$ pixel mesh with the unit cell tiled to form a supercell approximately $42 \times 37 \AA$. A total 80 Monte Carlo passes were used. A probe forming aperture of $30 \mathrm{mrad}$ was assumed.

Experimental 4D imaging was acquired using a Nion UltraSTEM 100 microscope operated at the $60 \mathrm{kV}$ accelerating voltage and the $30 \mathrm{mrad}$ convergence angle. The pixel dwell time was $20 \mathrm{~ms}$.

CVD-grown graphene samples were transferred from $\mathrm{Cu}$ foil to TEM grids and cleaned via a wet transfer method and baking in an $\mathrm{ArO}_{2}$ environment as described elsewhere. ${ }^{40}$ The dopant atom was inserted in situ as described elsewhere. ${ }^{41}$

The spectral clustering algorithm has been included in the Python sklearn package at http://scikit-learn.org/stable. The HDBSCAN Python package can be found at https://github.com/scikit-learn-contrib/hdbscan. The UMAP Python package can be found at https://github.com/Imcinnes/ umap.

\section{DATA AVAILABILITY}

The data that supports the findings of this study are available at https://doi.org/ 10.6084/m9.figshare.7416317. Python scripts and the GUI based on Python Bokeh library are available at https://github.com/nonmin/4D-STEM.

\section{ACKNOWLEDGEMENTS}

This research was supported by the US Department of Energy, Basic Energy Sciences, Materials Sciences and Engineering Division (M.P.O., A.R.L., S.V.K.) and conducted at the Center for Nanophase Materials Sciences, which is a US DOE Office of Science User Facility (X.L., O.E.D., S.J.). L.M. and J.H. acknowledge support from Tutte Institute for Mathematics and Computing, Canada. We gratefully acknowledge Myron D. Kapetanakis for providing the structure files used in the simulation.

\section{AUTHOR CONTRIBUTIONS}

X.L., S.J., and S.V.K. conceived and designed the study. S.J. and S.V.K. supervised the study. X.L. performed associated data analysis. O.E.D. synthesized material and carried out 4D-STEM experiments. M.P.O. performed simulation of Ronchigram datasets. A.R. L. contributed to synthetic and experimental data collection. X.L., L.M., and J.H. analyzed manifold learning algorithms. X.L. and S.V.K. co-wrote the article with comments from all authors.

\section{ADDITIONAL INFORMATION}

Supplementary information accompanies the paper on the npj Computational Materials website (https://doi.org/10.1038/s41524-018-0139-y).

Competing interests: The authors declare no competing interests.

Publisher's note Springer Nature remains neutral with regard to jurisdictional claims in published maps and institutional affiliations.

\section{REFERENCES}

1. Yang, Y. et al. Deciphering chemical order/disorder and material properties at the single-atom level. Nature 542, 75-79 (2017).

2. Yang, H. et al. Simultaneous atomic-resolution electron ptychography and Zcontrast imaging of light and heavy elements in complex nanostructures. Nat. Commun. 7, 12532 (2016)

3. Ophus, C. et al. Efficient linear phase contrast in scanning transmission electron microscopy with matched illumination and detector interferometry. Nat. Commun. 7, 10719 (2016).

4. Gammer, C. et al. Local and transient nanoscale strain mapping during in situ deformation. Appl. Phys. Lett. 109, 081906 (2016).

5. Pennycook, T. J. et al. Efficient phase contrast imaging in STEM using a pixelated detector. Part 1: Experimental demonstration at atomic resolution. Ultramicroscopy 151, 160-167 (2015).

6. Rodenburg, J. M. Ptychography and related diffractive imaging methods. Adv. Imag. Electron Phys. 150, 87-184 (2008).

7. Humphry, M. J., Kraus, B., Hurst, A. C., Maiden, A. M. \& Rodenburg, J. M. Ptychographic electron microscopy using high-angle dark-field scattering for subnanometre resolution imaging. Nat. Commun. 3, 730 (2012).

8. Gao, S. et al. Electron ptychographic microscopy for three-dimensional imaging. Nat. Commun. 8, 163 (2017).

9. Latychevskaia, T., Hsu, W.-H., Chang, W.-T., Lin, C.-Y. \& Hwang, I.-S. Threedimensional surface topography of graphene by divergent beam electron diffraction. Nat. Commun. 8, 14440 (2017).

10. Latychevskaia, T. et al. Convergent beam electron holography for analysis of van der Waals heterostructures. Proc. Natl Acad. Sci. USA 115, 7473-7478 (2018).

11. Rose, H. Phase contrast in scanning transmission electron microscopy. Opt (Stuttg.). 39, 416-436 (1974).

12. Dekkers, N. H. \& de Lang, H. Differential phase contrast in a stem. Opt. (Stuttg.). 41, 452-456 (1974).

13. Rose, $\mathrm{H}$. Nonstandard imaging methods in electron microscopy. Ultramicroscopy 2, 251-267 (1977).

14. Chapman, J. N. The investigation of magnetic domain structures in thin foils by electron microscopy. J. Phys. D Appl. Phys. 17, 623-647 (1984).

15. Chapman, J. N., McFadyen, I. R. \& McVitie, S. Modified differential phase contrast Lorentz microscopy for improved imaging of magnetic structures. IEEE Trans. Magn. 26, 1506-1511 (1990).

16. Lohr, M. et al. Differential phase contrast 2.0-Opening new "fields" for an established technique. Ultramicroscopy 117, 7-14 (2012).

17. Shibata, N. et al. Imaging of built-in electric field at a $\mathrm{p}-\mathrm{n}$ junction by scanning transmission electron microscopy. Sci. Rep. 5, 10040 (2015).

18. Matsumoto, T. et al. Direct observation of 7 domain boundary core structure in magnetic skyrmion lattice. Sci. Adv. 2, e1501280-e1501280 (2016).

19. Shibata, N. et al. New area detector for atomic-resolution scanning transmission electron microscopy. J. Electron. Microsc. (Tokyo) 59, 473-479 (2010).

20. Shibata, N. et al. Differential phase-contrast microscopy at atomic resolution. Nat. Phys. 8, 611-615 (2012).

21. Shibata, N. et al. Electric field imaging of single atoms. Nat. Commun. 8, 15631 (2017).

22. Müller, K. et al. Atomic electric fields revealed by a quantum mechanical approach to electron picodiffraction. Nat. Commun. 5, 5653 (2014).

23. Jesse, S. et al. Big data analytics for scanning transmission electron microscopy ptychography. Sci. Rep. 6, 26348 (2016).

24. McInnes, L. \& Healy, J. UMAP: Uniform Manifold Approximation and Projection for Dimension Reduction. arXiv preprint arXiv:1802.03426 (2018).

25. Tang, J., Liu, J., Zhang, M. \& Mei, Q. Visualizing Large-scale and High-dimensional Data. In Proc. 25th International Conference on World Wide Web (International World Wide Web Conference Committee, 2016). https://doi.org/10.1145/ 2872427.2883041.

26. Dasgupta, S. \& Freund, Y. Random projection trees and low dimensional manifolds. Proc. fortieth annu. ACM symp. Theory Comput., 6, 537-546 (2008).

27. Dong, W., Moses, C. \& Li, K. Efficient k-nearest neighbor graph construction for generic similarity measures. Proc. 20th Int. Conf. World wide web 11, 577 (2011) 
28. Mikolov, T., Chen, K., Corrado, G. \& Dean, J. Distributed Representations of Words and Phrases and their Compositionality. Adv. Neural Inf. Process. Syst. 3111-3119 (2013).

29. Li, X. et al. High-veracity functional imaging in scanning probe microscopy via Graph-Bootstrapping. Nat. Commun. 9, 2428 (2018).

30. Yu \& Shi. Multiclass spectral clustering. in Proc. Ninth IEEE International Conference on Computer Vision 313-319 vol.1 (IEEE, 2003). https://doi.org/10.1109/ ICCV.2003.1238361

31. Mclnnes, L., Healy, J. \& Astels, S. hdbscan: hierarchical density based clustering. J. Open Source Softw. 2, 205 (2017)

32. Campello, R. J. G. B., Moulavi, D., Zimek, A. \& Sander, J. Hierarchical density estimates for data clustering, visualization, and outlier detection. ACM Trans. Knowl. Discov. Data 10, 1-51 (2015).

33. Kalinin, S. V., Borisevich, A. \& Jesse, S. Fire up the atom forge. Nature 539, 485-487 (2016).

34. Dyck, O., Kim, S., Kalinin, S. V. \& Jesse S. E-beam manipulation of Si atoms on graphene edges with an aberration-corrected scanning transmission electron microscope. Nano Res. 11, 6217-6226 (2018).

35. Susi, T., Meyer, J. C. \& Kotakoski, J. Manipulating low-dimensional materials down to the level of single atoms with electron irradiation. Ultramicroscopy 180, 163-172 (2017).

36. Tripathi, M. et al. Electron-beam manipulation of silicon dopants in graphene. Nano Lett. 18, 5319-5232 (2018).

37. Hudak, B. M. et al. Directed atom-by-atom assembly of dopants in silicon. ACS Nano 12, 5873-5879 (2018).
38. Jesse, S. et al. Direct atomic fabrication and dopant positioning in Si using electron beams with active real-time image-based feedback. Nanotechnology 29, 255303 (2018).

39. Allen, L. J., D'Alfonso, A. J. \& Findlay, S. D. Modelling the inelastic scattering of fast electrons. Ultramicroscopy 151, 11-22 (2015).

40. Dyck, O., Kim, S., Kalinin, S. V. \& Jesse, S. Mitigating e-beam-induced hydrocarbon deposition on graphene for atomic-scale scanning transmission electron microscopy studies. J. Vac. Sci. Technol. B 36, 011801 (2018).

41. Dyck, O., Kim, S., Kalinin, S. V. \& Jesse, S. Placing single atoms in graphene with a scanning transmission electron microscope. Appl. Phys. Lett. 111, 113104 (2017).

(i) Open Access This article is licensed under a Creative Commons adaptation, distribution and reproduction in any medium or format, as long as you give appropriate credit to the original author(s) and the source, provide a link to the Creative Commons license, and indicate if changes were made. The images or other third party material in this article are included in the article's Creative Commons license, unless indicated otherwise in a credit line to the material. If material is not included in the article's Creative Commons license and your intended use is not permitted by statutory regulation or exceeds the permitted use, you will need to obtain permission directly from the copyright holder. To view a copy of this license, visit http://creativecommons. org/licenses/by/4.0/.

(c) This is a U.S. government work and not under copyright protection in the U.S.; foreign copyright protection may apply 2019 